\title{
Medicines Australia Code of Conduct: breaches
}

The Medicines Australia Code of Conduct guides the promotion of prescription products by pharmaceutical companies. ${ }^{1}$ Each year Medicines Australia publishes a report, from its Code of Conduct Committee, which details all the complaints that have been received about advertising and other promotional activities.

The latest report ${ }^{2}$ reflects the first year of operation of the 16th edition of the Code. As usual, most complaints about promotional activity were made by rival pharmaceutical companies or the Medicines Australia Monitoring Committee. Only about half of the complaints resulted in a breach of the Code being found. Table 1 shows the complaints where at least one breach was identified and more details can be found in the full report. ${ }^{2}$

\section{References}

1. Medicines Australia. Code of Conduct. 16th ed. 2009. www.medicinesaustralia.com.au [cited 2010 Nov 16]

2. Medicines Australia. Code of Conduct Annual Report 2010. www.medicinesaustralia.com.au [cited 2010 Nov 16]

\section{Table 1}

Breaches of the Code of Conduct July 2009 - June 2010

\begin{tabular}{|c|c|c|c|}
\hline Company & Brand (generic) name & Material or activity & Sanction imposed by Code of Conduct Committee \\
\hline Allergan & Botox (botulinum toxin) & Promotional material & $\begin{array}{l}\$ 100000 \text { fine } \\
\text { Withdraw material } \\
\text { Corrective letter }\end{array}$ \\
\hline Biogen Idec & Tysabri (natalizumab) & Promotional material & $\begin{array}{l}\$ 75000 \text { fine } \\
\text { Withdraw material } \\
\text { Corrective advertisement }\end{array}$ \\
\hline $\begin{array}{l}\text { Boehringer } \\
\text { Ingelheim }\end{array}$ & Mobic (meloxicam) & Advertorial & $\begin{array}{l}\$ 50000 \text { fine } \\
\text { Corrective advertisement }\end{array}$ \\
\hline \multirow[t]{2}{*}{ CSL } & $\begin{array}{l}\text { Human papillomavirus } \\
\text { vaccine }\end{array}$ & Information for the public & $\$ 1000$ fine \\
\hline & Various & Starter packs & $\$ 1000$ fine \\
\hline Genzyme & Renagel (sevelamer) & Patient education leaflet & $\begin{array}{l}\$ 25000 \text { fine } \\
\text { Withdraw material } \\
\text { Corrective letter }\end{array}$ \\
\hline GlaxoSmithKline & Valtrex (valaciclovir) & Advertising to the public & $\$ 150000$ fine reduced to $\$ 20000$ on appeal \\
\hline \multirow{3}{*}{$\begin{array}{l}\text { Merck Sharp \& } \\
\text { Dohme }\end{array}$} & - & Hospitality for specialists & $\$ 20000$ fine \\
\hline & - & Hospitality for specialists & $\$ 40000$ fine \\
\hline & - & Hospitality for specialists & $\$ 50000$ fine reduced to $\$ 10000$ on appeal \\
\hline Pharmalink & Pletal (cilostazol) & $\begin{array}{l}\text { Hospitality for specialists } \\
\text { and general practitioners }\end{array}$ & $\$ 50000$ fine \\
\hline \multirow[t]{2}{*}{ sanofi-aventis } & Copaxone (glatiramer) & Promotional material & $\begin{array}{l}\$ 25000 \text { fine } \\
\text { Withdraw material } \\
\text { Corrective letter }\end{array}$ \\
\hline & Clexane (enoxaparin) & Promotional material & Corrective letter \\
\hline Schering Plough & Olmetec (olmesartan) & Promotional material & $\begin{array}{l}\$ 35000 \text { fine } \\
\text { Withdraw material }\end{array}$ \\
\hline Servier & Coversyl (perindopril) & Promotional material & $\begin{array}{l}\$ 100000 \text { fine } \\
\text { Withdraw material } \\
\text { Corrective letter } \\
\text { (Requirement for corrective advertisement } \\
\text { removed on appeal) }\end{array}$ \\
\hline
\end{tabular}

